\title{
SMOOTH HYPERSURFACE SECTIONS CONTAINING A GIVEN SUBSCHEME OVER A FINITE FIELD
}

\author{
BJORN POONEN
}

\section{Introduction}

Let $\mathbb{F}_{q}$ be a finite field of $q=p^{a}$ elements. Let $X$ be a smooth quasi-projective subscheme of $\mathbb{P}^{n}$ of dimension $m \geq 0$ over $\mathbb{F}_{q}$. N. Katz asked for a finite field analogue of the Bertini smoothness theorem, and in particular asked whether one could always find a hypersurface $H$ in $\mathbb{P}^{n}$ such that $H \cap X$ is smooth of dimension $m-1$. A positive answer was proved in Gab01 and $\mathrm{Poo04}$ independently. The latter paper proved also that in a precise sense, a positive fraction of hypersurfaces have the required property.

The classical Bertini theorem was extended in Blo70, KA79 to show that the hypersurface can be chosen so as to contain a prescribed closed smooth subscheme $Z$, provided that the condition $\operatorname{dim} X>2 \operatorname{dim} Z$ is satisfied. (The condition arises naturally from a dimension-counting argument.) The goal of the current paper is to prove an analogous result over finite fields. In fact, our result is stronger than that of KA79 in that we do not require $Z \subseteq X$, but weaker in that we assume that $Z \cap X$ be smooth. (With a little more work and complexity, we could prove a version for a non-smooth intersection as well, but we restrict to the smooth case for simplicity.) One reason for proving our result is that it is used by SS07.

Let $S=\mathbb{F}_{q}\left[x_{0}, \ldots, x_{n}\right]$ be the homogeneous coordinate ring of $\mathbb{P}^{n}$. Let $S_{d} \subseteq S$ be the $\mathbb{F}_{q}$-subspace of homogeneous polynomials of degree $d$. For each $f \in S_{d}$, let $H_{f}$ be the subscheme $\operatorname{Proj}(S /(f)) \subseteq \mathbb{P}^{n}$. For the rest of this paper, we fix a closed subscheme $Z \subseteq \mathbb{P}^{n}$. For $d \in \mathbb{Z}_{\geq 0}$, let $I_{d}$ be the $\mathbb{F}_{q}$-subspace of $f \in S_{d}$ that vanish on $Z$. Let $I_{\text {homog }}=\bigcup_{d \geq 0} I_{d}$. We want to measure the density of subsets of $I_{\text {homog }}$, but

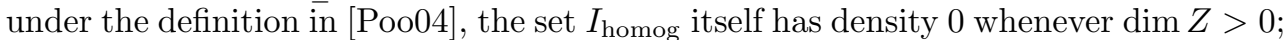
therefore we use a new definition of density, relative to $I_{\text {homog. Namely, we define the }}$ density of a subset $\mathcal{P} \subseteq I_{\text {homog }}$ by

$$
\mu_{Z}(\mathcal{P}):=\lim _{d \rightarrow \infty} \frac{\#\left(\mathcal{P} \cap I_{d}\right)}{\# I_{d}}
$$

if the limit exists. For a scheme $X$ of finite type over $\mathbb{F}_{q}$, define the zeta function Wei49

$$
\zeta_{X}(s)=Z_{X}\left(q^{-s}\right):=\prod_{\operatorname{closed} P \in X}\left(1-q^{-s \operatorname{deg} P}\right)^{-1}=\exp \left(\sum_{r=1}^{\infty} \frac{\# X\left(\mathbb{F}_{q^{r}}\right)}{r} q^{-r s}\right) ;
$$

Received by the editors March 8, 2007.

1991 Mathematics Subject Classification. Primary 14J70; Secondary 11M38, 11M41, 14G40, 14 N05.

This research was supported by NSF grant DMS-0301280. 
the product and sum converge when $\operatorname{Re}(s)>\operatorname{dim} X$.

Theorem 1.1. Let $X$ be a smooth quasi-projective subscheme of $\mathbb{P}^{n}$ of dimension $m \geq 0$ over $\mathbb{F}_{q}$. Let $Z$ be a closed subscheme of $\mathbb{P}^{n}$. Assume that the scheme-theoretic intersection $V:=Z \cap X$ is smooth of dimension $\ell . \quad($ If $V$ is empty, take $\ell=-1$.) Define

$$
\mathcal{P}:=\left\{f \in I_{\text {homog }}: H_{f} \cap X \text { is smooth of dimension } m-1\right\} .
$$

(i) If $m>2 \ell$, then

$$
\mu_{Z}(\mathcal{P})=\frac{\zeta_{V}(m+1)}{\zeta_{V}(m-\ell) \zeta_{X}(m+1)}=\frac{1}{\zeta_{V}(m-\ell) \zeta_{X-V}(m+1)} .
$$

In this case, in particular, for $d \gg 1$, there exists a degree-d hypersurface $H$ containing $Z$ such that $H \cap X$ is smooth of dimension $m-1$.

(ii) If $m \leq 2 \ell$, then $\mu_{Z}(\mathcal{P})=0$.

The proof will use the closed point sieve introduced in Poo04. In fact, the proof is parallel to the one in that paper, but changes are required in almost every line.

\section{Singular points of low degree}

Let $\mathcal{I}_{Z} \subseteq \mathcal{O}_{\mathbb{P}^{n}}$ be the ideal sheaf of $Z$, so $I_{d}=H^{0}\left(\mathbb{P}^{n}, \mathcal{I}_{Z}(d)\right)$. Tensoring the surjection

$$
\begin{aligned}
\mathcal{O}^{\oplus(n+1)} & \rightarrow \mathcal{O} \\
\left(f_{0}, \ldots, f_{n}\right) & \mapsto x_{0} f_{0}+\cdots+x_{n} f_{n}
\end{aligned}
$$

with $\mathcal{I}_{Z}$, twisting by $\mathcal{O}(d)$, and taking global sections shows that $S_{1} I_{d}=I_{d+1}$ for $d \gg 1$. Fix $c$ such that $S_{1} I_{d}=I_{d+1}$ for all $d \geq c$.

Before proving the main result of this section (Lemma 2.3), we need two lemmas.

Lemma 2.1. Let $Y$ be a finite subscheme of $\mathbb{P}^{n}$. Let

$$
\phi_{d}: I_{d}=H^{0}\left(\mathbb{P}^{n}, \mathcal{I}_{Z}(d)\right) \rightarrow H^{0}\left(Y, \mathcal{I}_{Z} \cdot \mathcal{O}_{Y}(d)\right)
$$

be the map induced by the map of sheaves $\mathcal{I}_{Z} \rightarrow \mathcal{I}_{Z} \cdot \mathcal{O}_{Y}$ on $\mathbb{P}^{n}$. Then $\phi_{d}$ is surjective for $d \geq c+\operatorname{dim} H^{0}\left(Y, \mathcal{O}_{Y}\right)$,

Proof. The map of sheaves $\mathcal{O}_{\mathbb{P}^{n}} \rightarrow \mathcal{O}_{Y}$ on $\mathbb{P}^{n}$ is surjective so $\mathcal{I}_{Z} \rightarrow \mathcal{I}_{Z} \cdot \mathcal{O}_{Y}$ is surjective too. Thus $\phi_{d}$ is surjective for $d \gg 1$.

Enlarging $\mathbb{F}_{q}$ if necessary, we can perform a linear change of variable to assume $Y \subseteq \mathbb{A}^{n}:=\left\{x_{0} \neq 0\right\}$. Dehomogenization (setting $x_{0}=1$ ) identifies $S_{d}$ with the space $S_{d}^{\prime}$ of polynomials in $\mathbb{F}_{q}\left[x_{1}, \ldots, x_{n}\right]$ of total degree $\leq d$. and identifies $\phi_{d}$ with a map

$$
I_{d}^{\prime} \rightarrow B:=H^{0}\left(\mathbb{P}^{n}, \mathcal{I}_{Z} \cdot \mathcal{O}_{Y}\right) \text {. }
$$

By definition of $c$, we have $S_{1}^{\prime} I_{d}^{\prime}=I_{d+1}^{\prime}$ for $d \geq c$. For $d \geq b$, let $B_{d}$ be the image of $I_{d}^{\prime}$ in $B$, so $S_{1}^{\prime} B_{d}=B_{d+1}$ for $d \geq c$. Since $1 \in S_{1}^{\prime}$, we have $I_{d}^{\prime} \subseteq I_{d+1}^{\prime}$, so

$$
B_{c} \subseteq B_{c+1} \subseteq \cdots .
$$

But $b:=\operatorname{dim} B<\infty$, so $B_{j}=B_{j+1}$ for some $j \in[c, c+b]$. Then

$$
B_{j+2}=S_{1}^{\prime} B_{j+1}=S_{1}^{\prime} B_{j}=B_{j+1} .
$$

Similarly $B_{j}=B_{j+1}=B_{j+2}=\ldots$, and these eventually equal $B$ by the previous paragraph. Hence $\phi_{d}$ is surjective for $d \geq j$, and in particular for $d \geq c+b$. 
Lemma 2.2. Suppose $\mathfrak{m} \subseteq \mathcal{O}_{X}$ is the ideal sheaf of a closed point $P \in X$. Let $Y \subseteq X$ be the closed subscheme whose ideal sheaf is $\mathfrak{m}^{2} \subseteq \mathcal{O}_{X}$. Then for any $d \in \mathbb{Z}_{\geq 0}$.

$$
\# H^{0}\left(Y, \mathcal{I}_{Z} \cdot \mathcal{O}_{Y}(d)\right)= \begin{cases}q^{(m-\ell) \operatorname{deg} P}, & \text { if } P \in V, \\ q^{(m+1) \operatorname{deg} P}, & \text { if } P \notin V .\end{cases}
$$

Proof. Since $Y$ is finite, we may now ignore the twisting by $\mathcal{O}(d)$. The space $H^{0}\left(Y, \mathcal{O}_{Y}\right)$ has a two-step filtration whose quotients have dimensions 1 and $m$ over the residue field $\kappa$ of $P$. Thus $\# H^{0}\left(Y, \mathcal{O}_{Y}\right)=(\# \kappa)^{m+1}=q^{(m+1) \operatorname{deg} P}$. If $P \in V$ (or equivalently $P \in Z$ ), then $H^{0}\left(Y, \mathcal{O}_{Z \cap Y}\right)$ has a filtration whose quotients have dimensions 1 and $\ell$ over $\kappa$; if $P \notin V$, then $H^{0}\left(Y, \mathcal{O}_{Z \cap Y}\right)=0$. Taking cohomology of

$$
0 \rightarrow \mathcal{I}_{Z} \cdot \mathcal{O}_{Y} \rightarrow \mathcal{O}_{Y} \rightarrow \mathcal{O}_{Z \cap Y} \rightarrow 0
$$

on the 0-dimensional scheme $Y$ yields

$$
\begin{aligned}
\# H^{0}\left(Y, \mathcal{I}_{Z} \cdot \mathcal{O}_{Y}\right) & =\frac{\# H^{0}\left(Y, \mathcal{O}_{Y}\right)}{\# H^{0}\left(Y, \mathcal{O}_{Z \cap Y}\right)} \\
& = \begin{cases}q^{(m+1) \operatorname{deg} P} / q^{(\ell+1) \operatorname{deg} P}, & \text { if } P \in V, \\
q^{(m+1) \operatorname{deg} P}, & \text { if } P \notin V .\end{cases}
\end{aligned}
$$

If $U$ is a scheme of finite type over $\mathbb{F}_{q}$, let $U_{<r}$ be the set of closed points of $U$ of degree $<r$. Similarly define $U_{>r}$.

Lemma 2.3 (Singularities of low degree). Let notation and hypotheses be as in Theorem 1.1, and define

$$
\mathcal{P}_{r}:=\left\{f \in I_{\text {homog }}: H_{f} \cap X \text { is smooth of dimension } m-1 \text { at all } P \in X_{<r}\right\} .
$$

Then

$$
\mu_{Z}\left(\mathcal{P}_{r}\right)=\prod_{P \in V_{<r}}\left(1-q^{-(m-\ell) \operatorname{deg} P}\right) \cdot \prod_{P \in(X-V)<r}\left(1-q^{-(m+1) \operatorname{deg} P}\right) .
$$

Proof. Let $X_{<r}=\left\{P_{1}, \ldots, P_{s}\right\}$. Let $\mathfrak{m}_{i}$ be the ideal sheaf of $P_{i}$ on $X$. let $Y_{i}$ be the closed subscheme of $X$ with ideal sheaf $\mathfrak{m}_{i}^{2} \subseteq \mathcal{O}_{X}$, and let $Y=\bigcup Y_{i}$. Then $H_{f} \cap X$ is singular at $P_{i}$ (more precisely, not smooth of dimension $m-1$ at $P_{i}$ ) if and only if the restriction of $f$ to a section of $\mathcal{O}_{Y_{i}}(d)$ is zero.

By Lemma 2.1. $\mu_{Z}(\mathcal{P})$ equals the fraction of elements in $H^{0}\left(\mathcal{I}_{Z} \cdot \mathcal{O}_{Y}(d)\right)$ whose restriction to a section of $\mathcal{O}_{Y_{i}}(d)$ is nonzero for every $i$. Thus

$$
\begin{aligned}
\mu_{Z}\left(\mathcal{P}_{r}\right) & =\prod_{i=1}^{s} \frac{\# H^{0}\left(Y_{i}, \mathcal{I}_{Z} \cdot \mathcal{O}_{Y_{i}}\right)-1}{\# H^{0}\left(Y_{i}, \mathcal{I}_{Z} \cdot \mathcal{O}_{Y_{i}}\right)} \\
& =\prod_{P \in V_{<r}}\left(1-q^{-(m-\ell) \operatorname{deg} P}\right) \cdot \prod_{P \in(X-V)_{<r}}\left(1-q^{-(m+1) \operatorname{deg} P}\right),
\end{aligned}
$$

by Lemma 2.2 . 
Corollary 2.4. If $m>2 \ell$, then

$$
\lim _{r \rightarrow \infty} \mu_{Z}\left(\mathcal{P}_{r}\right)=\frac{\zeta_{V}(m+1)}{\zeta_{X}(m+1) \zeta_{V}(m-\ell)} .
$$

Proof. The products in Lemma 2.3 are the partial products in the definition of the zeta functions. For convergence, we need $m-\ell>\operatorname{dim} V=\ell$, which is equivalent to $m>2 \ell$.

Proof of Theorem 1.1 (ii). We have $\mathcal{P} \subseteq \mathcal{P}_{r}$. By Lemma 2.3.

$$
\mu_{Z}\left(\mathcal{P}_{r}\right) \leq \prod_{P \in V_{<r}}\left(1-q^{-(m-\ell) \operatorname{deg} P}\right)
$$

which tends to 0 as $r \rightarrow \infty$ if $m \leq 2 \ell$. Thus $\mu_{Z}(\mathcal{P})=0$ in this case.

From now on, we assume $m>2 \ell$.

\section{Singular points of medium degree}

Lemma 3.1. Let $P \in X$ is a closed point of degree $e$, where $e \leq \frac{d-c}{m+1}$. Then the fraction of $f \in I_{d}$ such that $H_{f} \cap X$ is not smooth of dimension $m-1$ at $P$ equals

$$
\begin{cases}q^{-(m-\ell) e}, & \text { if } P \in V, \\ q^{-(m+1) e}, & \text { if } P \notin V .\end{cases}
$$

Proof. This follows by applying Lemma 2.1 to the $Y$ in Lemma 2.2. and then applying Lemma 2.2

Define the upper and lower densities $\bar{\mu}_{Z}(\mathcal{P}), \underline{\mu}_{Z}(\mathcal{P})$ of a subset $\mathcal{P} \subseteq I_{\text {homog }}$ as $\mu_{Z}(\mathcal{P})$ was defined, but using limsup and lim inf in place of lim.

Lemma 3.2 (Singularities of medium degree). Define

$$
\begin{aligned}
\mathcal{Q}_{r}^{\text {medium }}:=\bigcup_{d \geq 0}\left\{f \in I_{d} \text { : there exists } P \in X \text { with } r \leq \operatorname{deg} P \leq \frac{d-b}{m+1}\right. \\
\text { such that } \left.H_{f} \cap X \text { is not smooth of dimension } m-1 \text { at } P\right\} .
\end{aligned}
$$

Then $\lim _{r \rightarrow \infty} \bar{\mu}_{Z}\left(\mathcal{Q}_{r}^{\text {medium }}\right)=0$.

Proof. By Lemma 3.1, we have

$$
\begin{aligned}
\frac{\#\left(\mathcal{Q}_{r}^{\text {medium }} \cap I_{d}\right)}{\# I_{d}} & \leq \sum_{\substack{P \in Z \\
r \leq \operatorname{deg} P \leq \frac{d-b}{m+1}}} q^{-(m-\ell) \operatorname{deg} P}+\sum_{\substack{P \in X-Z \\
r \leq \operatorname{deg} P \leq \frac{d-b}{m+1}}} q^{-(m+1) \operatorname{deg} P} \\
& \leq \sum_{P \in Z_{\geq r}} q^{-(m-\ell) \operatorname{deg} P}+\sum_{P \in(X-Z)_{\geq r}} q^{-(m+1) \operatorname{deg} P .}
\end{aligned}
$$

Using the trivial bound that an $m$-dimensional variety has at most $O\left(q^{e m}\right)$ closed points of degree $e$, as in the proof of Poo04, Lemma 2.4], we show that each of the two sums converges to a value that is $O\left(q^{-r}\right)$ as $r \rightarrow \infty$, under our assumption $m>2 \ell$. 


\section{Singular points of high degree}

Lemma 4.1. Let $P$ be a closed point of degree $e$ in $\mathbb{P}^{n}-Z$. For $d \geq c$, the fraction of $f \in I_{d}$ that vanish at $P$ is at most $q^{-\min (d-c, e)}$.

Proof. Equivalently, we must show that the image of $\phi_{d}$ in Lemma 2.1 for $Y=P$ has $\mathbb{F}_{q}$-dimension at least $\min (d-c, e)$. The proof of Lemma 2.1 shows that as $d$ runs through the integers $c, c+1, \ldots$, this dimension increases by at least 1 until it reaches its maximum, which is $e$.

Lemma 4.2 (Singularities of high degree off $V$ ). Define

$$
\begin{aligned}
& \mathcal{Q}_{X-V}^{\text {high }}:=\bigcup_{d \geq 0}\left\{f \in I_{d}: \exists P \in(X-V)_{>\frac{d-c}{m+1}}\right. \\
&\text { such that } \left.H_{f} \cap X \text { is not smooth of dimension } m-1 \text { at } P\right\}
\end{aligned}
$$

Then $\bar{\mu}_{Z}\left(\mathcal{Q}_{X-V}^{\text {high }}\right)=0$.

Proof. It suffices to prove the lemma with $X$ replaced by each of the sets in an open covering of $X-V$, so we may assume $X$ is contained in $\mathbb{A}^{n}=\left\{x_{0} \neq 0\right\} \subseteq \mathbb{P}^{n}$, and that $V=\emptyset$. Dehomogenize by setting $x_{0}=1$, to identify $I_{d} \subseteq S_{d}$ with subspaces of $I_{d}^{\prime} \subseteq S_{d}^{\prime} \subseteq A:=\mathbb{F}_{q}\left[x_{1}, \ldots, x_{n}\right]$.

Given a closed point $x \in X$, choose a system of local parameters $t_{1}, \ldots, t_{n} \in A$ at $x$ on $\mathbb{A}^{n}$ such that $t_{m+1}=t_{m+2}=\cdots=t_{n}=0$ defines $X$ locally at $x$. Multiplying all the $t_{i}$ by an element of $A$ vanishing on $Z$ but nonvanishing at $x$, we may assume in addition that all the $t_{i}$ vanish on $Z$. Now $d t_{1}, \ldots, d t_{n}$ are a $\mathcal{O}_{\mathbb{A}^{n}, x^{-}}$-basis for the stalk $\Omega_{\mathbb{A}^{n} / \mathbb{F}_{q}, x}^{1}$. Let $\partial_{1}, \ldots, \partial_{n}$ be the dual basis of the stalk $\mathcal{T}_{\mathbb{A}^{n} / \mathbb{F}_{q}, x}$ of the tangent sheaf. Choose $s \in A$ with $s(x) \neq 0$ to clear denominators so that $D_{i}:=s \partial_{i}$ gives a global derivation $A \rightarrow A$ for $i=1, \ldots, n$. Then there is a neighborhood $N_{x}$ of $x$ in $\mathbb{A}^{n}$ such that $N_{x} \cap\left\{t_{m+1}=t_{m+2}=\cdots=t_{n}=0\right\}=N_{x} \cap X, \Omega_{N_{x} / \mathbb{F}_{q}}^{1}=\oplus_{i=1}^{n} \mathcal{O}_{N_{x}} d t_{i}$, and $s \in \mathcal{O}\left(N_{u}\right)^{*}$. We may cover $X$ with finitely many $N_{x}$, so we may reduce to the case where $X \subseteq N_{x}$ for a single $x$. For $f \in I_{d}^{\prime} \simeq I_{d}, H_{f} \cap X$ fails to be smooth of dimension $m-1$ at a point $P \in U$ if and only if $f(P)=\left(D_{1} f\right)(P)=\cdots=\left(D_{m} f\right)(P)=0$.

Let $\tau=\max _{i}\left(\operatorname{deg} t_{i}\right), \gamma=\lfloor(d-\tau) / p\rfloor$, and $\eta=\lfloor d / p\rfloor$. If $f_{0} \in I_{d}^{\prime}, g_{1} \in S_{\gamma}^{\prime}, \ldots$, $g_{m} \in S_{\gamma}^{\prime}$, and $h \in I_{\eta}^{\prime}$ are selected uniformly and independently at random, then the distribution of

$$
f:=f_{0}+g_{1}^{p} t_{1}+\cdots+g_{m}^{p} t_{m}+h^{p}
$$

is uniform over $I_{d}^{\prime}$, because of $f_{0}$. We will bound the probability that an $f$ constructed in this way has a point $P \in X_{>\frac{d-c}{m+1}}$ where $f(P)=\left(D_{1} f\right)(P)=\cdots=\left(D_{m} f\right)(P)=0$. We have $D_{i} f=\left(D_{i} f_{0}\right)+g_{i}^{p} s$ for $i=1, \ldots, m$. We will select $f_{0}, g_{1}, \ldots, g_{m}, h$ one at a time. For $0 \leq i \leq m$, define

$$
W_{i}:=X \cap\left\{D_{1} f=\cdots=D_{i} f=0\right\} .
$$

Claim 1: For $0 \leq i \leq m-1$, conditioned on a choice of $f_{0}, g_{1}, \ldots, g_{i}$ for which $\operatorname{dim}\left(W_{i}\right) \leq m-i$, the probability that $\operatorname{dim}\left(W_{i+1}\right) \leq m-i-1$ is $1-o(1)$ as $d \rightarrow \infty$. (The function of $d$ represented by the $o(1)$ depends on $X$ and the $D_{i}$.)

Proof of Claim 1: This is completely analogous to the corresponding proof in Poo04]. 
Claim 2: Conditioned on a choice of $f_{0}, g_{1}, \ldots, g_{m}$ for which $W_{m}$ is finite, $\operatorname{Prob}\left(H_{f} \cap\right.$ $\left.W_{m} \cap X_{>\frac{d-c}{m+1}}=\emptyset\right)=1-o(1)$ as $d \rightarrow \infty$.

Proof of Claim 2: By Bézout's theorem as in [Ful84, p. 10], we have $\# W_{m}=O\left(d^{m}\right)$. For a given point $P \in W_{m}$, the set $H^{\text {bad }}$ of $h \in I_{\eta}^{\prime}$ for which $H_{f}$ passes through $P$ is either $\emptyset$ or a coset of $\operatorname{ker}\left(\operatorname{ev}_{P}: I_{\eta}^{\prime} \rightarrow \kappa(P)\right)$, where $\kappa(P)$ is the residue field of $P$, and $\mathrm{ev}_{P}$ is the evaluation-at- $P$ map. If moreover $\operatorname{deg} P>\frac{d-c}{m+1}$, then Lemma 4.1 implies $\# H^{\text {bad }} / \# I_{\eta}^{\prime} \leq q^{-\nu}$ where $\nu=\min \left(\eta, \frac{d-c}{m+1}\right)$. Hence

$$
\operatorname{Prob}\left(H_{f} \cap W_{m} \cap X_{>\frac{d-c}{m+1}} \neq \emptyset\right) \leq \# W_{m} q^{-\nu}=O\left(d^{m} q^{-\nu}\right)=o(1)
$$

as $d \rightarrow \infty$, since $\nu$ eventually grows linearly in $d$. This proves Claim 2 .

End of proof: Choose $f \in I_{d}$ uniformly at random. Claims 1 and 2 show that with probability $\prod_{i=0}^{m-1}(1-o(1)) \cdot(1-o(1))=1-o(1)$ as $d \rightarrow \infty, \operatorname{dim} W_{i}=m-i$ for $i=0,1, \ldots, m$ and $H_{f} \cap W_{m} \cap X_{>\frac{d-c}{m+1}}=\emptyset$. But $H_{f} \cap W_{m}$ is the subvariety of $X$ cut out by the equations $f(P)=\left(D_{1} f\right)(P)=\cdots=\left(D_{m} f\right)(P)=0$, so $H_{f} \cap W_{m} \cap X_{>\frac{d-c}{m+1}}$ is exactly the set of points of $H_{f} \cap X$ of degree $>\frac{d-c}{m+1}$ where $H_{f} \cap X$ is not smooth of dimension $m-1$. Thus $\bar{\mu}_{Z}\left(\mathcal{Q}_{X-V}^{\text {high }}\right)=0$.

Lemma 4.3 (Singularities of high degree on $V$ ). Define

$$
\begin{aligned}
& \mathcal{Q}_{V}^{\text {high }}:=\bigcup_{d \geq 0}\left\{f \in I_{d}: \exists P \in V_{>\frac{d-c}{m+1}}\right. \\
&\text { such that } \left.H_{f} \cap X \text { is not smooth of dimension } m-1 \text { at } P\right\} .
\end{aligned}
$$

Then $\bar{\mu}_{Z}\left(\mathcal{Q}_{V}^{\text {high }}\right)=0$.

Proof. As before, we may assume $X \subseteq \mathbb{A}^{n}$ and we may dehomogenize. Given a closed point $x \in X$, choose a system of local parameters $t_{1}, \ldots, t_{n} \in A$ at $x$ on $\mathbb{A}^{n}$ such that $t_{m+1}=t_{m+2}=\cdots=t_{n}=0$ defines $X$ locally at $x$, and $t_{1}=t_{2}=\cdots=t_{m-\ell}=$ $t_{m+1}=t_{m+2}=\cdots=t_{n}=0$ defines $V$ locally at $x$. If $\mathfrak{m}_{w}$ is the ideal sheaf of $w$ on $\mathbb{P}^{n}$, then $\mathcal{I}_{Z} \rightarrow \frac{\mathfrak{m}_{w}}{\mathfrak{m}^{2}}$ is surjective, so we may adjust $t_{1}, \ldots, t_{m-\ell}$ to assume that they vanish not only on $V$ but also on $Z$.

Define $\partial_{i}$ and $D_{i}$ as in the proof of Lemma 4.2. Then there is a neighborhood $N_{x}$ of $x$ in $\mathbb{A}^{n}$ such that $N_{x} \cap\left\{t_{m+1}=t_{m+2}=\cdots=t_{n}=0\right\}=N_{x} \cap X, \Omega_{N_{x} / \mathbb{F}_{q}}^{1}=$ $\oplus_{i=1}^{n} \mathcal{O}_{N_{x}} d t_{i}$, and $s \in \mathcal{O}\left(N_{u}\right)^{*}$. Again we may assume $X \subseteq N_{x}$ for a single $x$. For $f \in I_{d}^{\prime} \simeq I_{d}, H_{f} \cap X$ fails to be smooth of dimension $m-1$ at a point $P \in V$ if and only if $f(P)=\left(D_{1} f\right)(P)=\cdots=\left(D_{m} f\right)(P)=0$.

Again let $\tau=\max _{i}\left(\operatorname{deg} t_{i}\right), \gamma=\lfloor(d-\tau) / p\rfloor$, and $\eta=\lfloor d / p\rfloor$. If $f_{0} \in I_{d}^{\prime}, g_{1} \in S_{\gamma}^{\prime}$, $\ldots, g_{\ell+1} \in S_{\gamma}^{\prime}$, are chosen uniformly at random, then

$$
f:=f_{0}+g_{1}^{p} t_{1}+\cdots+g_{\ell+1}^{p} t_{\ell+1}
$$

is a random element of $I_{d}^{\prime}$, since $\ell+1 \leq m-\ell$.

For $i=0, \ldots, \ell+1$, the subscheme

$$
W_{i}:=V \cap\left\{D_{1} f=\cdots=D_{i} f=0\right\}
$$


depends only on the choices of $f_{0}, g_{1}, \ldots, g_{i}$. The same argument as in the previous proof shows that for $i=0, \ldots, \ell$, we have

$$
\operatorname{Prob}\left(\operatorname{dim} W_{i} \leq \ell-i\right)=1-o(1)
$$

as $d \rightarrow \infty$. In particular, $W_{\ell}$ is finite with probability $1-o(1)$.

To prove that $\bar{\mu}_{Z}\left(\mathcal{Q}_{V}^{\text {high }}\right)=0$, it remains to prove that conditioned on choices of $f_{0}, g_{1}, \ldots, g_{\ell}$ making $\operatorname{dim} W_{\ell}$ finite,

$$
\operatorname{Prob}\left(W_{\ell+1} \cap V_{>\frac{d-c}{m+1}}=\emptyset\right)=1-o(1) .
$$

By Bézout's theorem, $\# W_{\ell}=O\left(d^{\ell}\right)$. The set $H^{\text {bad }}$ of choices of $g_{\ell+1}$ making $D_{\ell+1} f$ vanish at a given point $P \in W_{\ell}$ is either empty or a coset of $\operatorname{ker}\left(\operatorname{ev}_{P}: S_{\gamma}^{\prime} \rightarrow \kappa(P)\right)$. Lemma 2.5 of Poo04 implies that the size of this kernel (or its coset) as a fraction of $\# S_{\gamma}^{\prime}$ is at most $q^{-\nu}$ where $\nu:=\min \left(\gamma, \frac{d-c}{m+1}\right)$. Since $\# W_{\ell} q^{\nu}=o(1)$ as $d \rightarrow \infty$, we are done.

\section{Conclusion}

Proof of Theorem 1.1(i). We have

$$
\mathcal{P} \subseteq \mathcal{P}_{r} \subseteq \mathcal{P} \cup \mathcal{Q}_{r}^{\text {medium }} \cup \mathcal{Q}_{X-V}^{\text {high }} \cup \mathcal{Q}_{V}^{\text {high }},
$$

so $\bar{\mu}_{Z}(\mathcal{P})$ and $\underline{\mu}_{Z}(\mathcal{P})$ each differ from $\mu_{Z}\left(\mathcal{P}_{r}\right)$ by at most $\bar{\mu}_{Z}\left(\mathcal{Q}_{r}^{\text {medium }}\right)+\bar{\mu}_{Z}\left(\mathcal{Q}_{X-V}^{\text {high }}\right)+$ $\bar{\mu}_{Z}\left(\mathcal{Q}_{V}^{\text {high }}\right)$. Applying Corollary 2.4 and Lemmas $3.2,4.2$ and 4.3 , we obtain

$$
\mu_{Z}(\mathcal{P})=\lim _{r \rightarrow \infty} \mu_{Z}\left(\mathcal{P}_{r}\right)=\frac{\zeta_{V}(m+1)}{\zeta_{V}(m-\ell) \zeta_{X}(m+1)} .
$$

\section{Acknowledgements}

I thank Shuji Saito for asking the question answered by this paper, and for pointing out [KA79].

\section{References}

[Blo70] S. Bloch, 1970. Ph.D. thesis, Columbia University.

[Ful84] W. Fulton, Introduction to intersection theory in algebraic geometry, CBMS Regional Conference Series in Mathematics, vol. 54, Published for the Conference Board of the Mathematical Sciences, Washington, DC, 1984.MR735435 (85j:14008)

[Gab01] O. Gabber, On space filling curves and Albanese varieties, Geom. Funct. Anal. 11 (2001), no. 6, 1192-1200.MR1878318 (2003g:14034)

[KA79] S. L. Kleiman and Allen B. Altman, Bertini theorems for hypersurface sections containing a subscheme, Comm. Algebra 7 (1979), no. 8, 775-790.MR529493 (81i:14007)

[Poo04] B. Poonen, Bertini theorems over finite fields, Ann. of Math. (2) 160 (2004), no. 3, 10991127. MR 2144974 (2006a:14035)

[SS07] S. Saito and Kanetomo Sato, Finiteness theorem on zero-cycles over p-adic fields (April 11, 2007). arXiv:math.AG/0605165.

[Wei49] A. Weil, Numbers of solutions of equations in finite fields, Bull. Amer. Math. Soc. 55 (1949), 497-508.MR0029393 (10,592e)

Department of Mathematics, University of California, Berkeley, CA 94720-3840, USA

E-mail address: poonen@math.berkeley.edu

URL: http://math.berkeley.edu/ ${ }^{\sim}$ poonen 